\title{
A four objectives optimization for an energy system considered in the environment
}

\author{
M. Blaise $\cdot$ M. Feidt
}

Received: 30 July 2014/ Accepted: 23 September 2014/Published online: 11 October 2014

(C) The Author(s) 2014. This article is published with open access at Springerlink.com

\begin{abstract}
To optimize a system, the two main points to consider are a precise system definition (boundary between the system and the environment) and a precise choice of criteria to fulfill. Various energy, environmental and economical goals imply various criteria to minimize energy consumption, rejected nonrecoverable heat or entropy production as well as a maximization of the useful effect (even the efficiency). We propose a generalized criterion that takes into account the main four objectives of an energy system. Weighting factors are allocated to the different objectives. To illustrate this point, we consider a Carnot engine in contact with two infinite heat reservoirs (thermostats). The heat transfer model considered uses linear transfer law (constant heat transfer conductance). Thermal losses are considered at various scales. The converter entropy production is supposed to be independent of its hot and cold temperatures that are the two optimization variables. Numerical optimization is performed with various weighting factor distributions. Limit cases are recovered and correspond to optimization results existing in the literature (mainly one-criterion optimizations). However, we distinguish clearly three fundamental assemblies that could be optimized: the converter in contact with the source and sink, the system composed of converter, source and sink and the same system placed in the environment.
\end{abstract}

M. Blaise $(\varangle) \cdot$ M. Feidt

Laboratoire d'Energétique et de Mécanique Théorique et Appliquée (LEMTA-ENSEM), 2, avenue de la Forêt de Haye, 54516 Vandœuvre-Lès-Nancy, France

e-mail: mathilde.blaise@univ-lorraine.fr

\section{Blaise}

Agence de l'Environnement et de la Maîtrise de l'Energie (ADEME), 20, avenue du Grésillé- BP 90406,

49004 Angers Cedex 01, France
Keywords Multiobjective optimization · Energy system · Environment $\cdot$ Entropy analysis

$\begin{array}{ll}\text { List of symbols } \\ \dot{Q} & \text { Heat flow rate (W) } \\ \dot{S} & \text { Entropy rate (W/K) } \\ \dot{W} & \text { Mechanical power }(\mathrm{W}) \\ K & \text { Heat transfer conductance }(\mathrm{W} / \mathrm{K}) \\ T & \text { Temperature }(\mathrm{K}) \\ q & \text { Dimensionless heat flow rate } \\ s & \text { Dimensionless entropy rate } \\ w & \text { Dimensionless mechanical power } \\ \mathrm{k} & \text { Dimensionless heat transfer conductance } \\ t & \text { Dimensionless temperature } \\ \eta & \text { First law efficiency } \\ \mathrm{OF} & \text { Objective function } \\ \alpha & \text { Mechanical power weighting factor } \\ \beta & \text { Heat expense weighting factor } \\ \gamma & \text { Heat rejection weighting factor } \\ \delta & \text { Entropy creation weighting factor }\end{array}$

\section{Subscripts}

$H, h \quad$ High value (hot side)

$L, l \quad$ Low value (cold side)

C Converter alone

$i \quad$ Converter with its thermal contacts

S System

S0 System in the environment

$l$ Loss

$T$ Total

0 Environment

\section{Superscripts}

* Optimum value 


\section{Introduction}

Many criteria exist for optimizing a given energy system. Figure 1 shows the principal ones, under the form of a triangular scheme. The three basic criteria are energy consumption (EC), rejected heat $\mathrm{R}$ (or heat pollution $\mathrm{P}$ ) and more frequently the energy useful effect (UE). Classically, equilibrium thermodynamics considers the first law efficiency $\eta_{\mathrm{I}}$. More recently, the minimum of entropy production was considered.

The first types of optimization performed in the past considered the maximization of the first law efficiency according to the Carnot equilibrium thermodynamics for the appraisal of an energy system. During the second part of the last century, maximization of the mechanical power of engines was considered. This appraisal was initiated by Chambadal [1-3] and Novikov [4] and renewed by Curzon and Alhborn [5]. These papers mark the beginning of the finite time thermodynamics, FTT. Today, the simultaneous consideration of energy, environment and economy concern implies a multicriterion analysis. Multicriterion optimization of thermal systems design considering simultaneously energy, economy and environment as objectives is developed [6]. These studies are developed for engines and plants, as well as for refrigeration systems [7] and for combined cooling, heating and power systems [8], even more at a national level [9]. New heuristic optimization methods (evolutionary algorithms) are also applied, for example to heat pump [10] or solar heat engine [11]. These algorithms appear strongly connected to the second law. A criterion combining the minimization of the entropy production and the maximization of the mechanical power output is proposed by Angulo-Brown [12] and completed by Yan [13]. It is valuable for the global system. The utilization of this kind of criteria is a means to obtain a compromise between power, cost and pollution objectives. In this paper, a new mixed objective is proposed, taking account of four aspects, which are power, cost, heat rejection and entropy production.

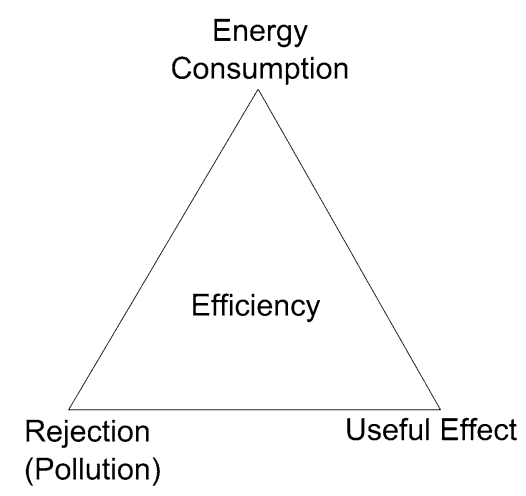

Fig. 1 The triangle of optimization criteria
A compromise between all of the criteria is difficult to consider. For example, the multiobjective Pareto approach can be used and defines an area of optimal trade-offs among all the objectives [6]. The four aspects considered in this paper are related through weighting factors. The compromise between each objective is therefore flexible. The weighting factor variation permits the observation of physically acceptable areas.

\section{Modeling of Novikov-Curzon-Ahlborn thermomechanical engine}

The model proposed here is an extended one (Fig. 2a) of the thermomechanical engine proposed initially by Novikov-Curzon-Ahlborn. This extended model takes

(a)

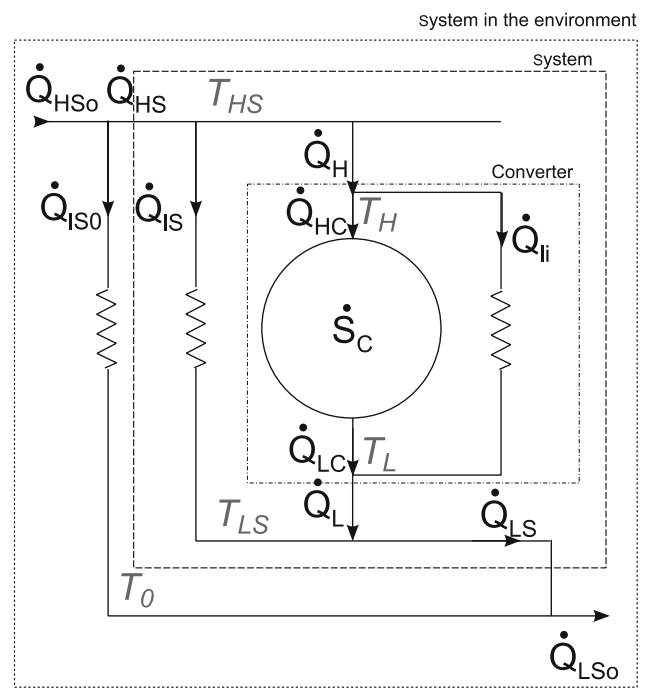

(b)

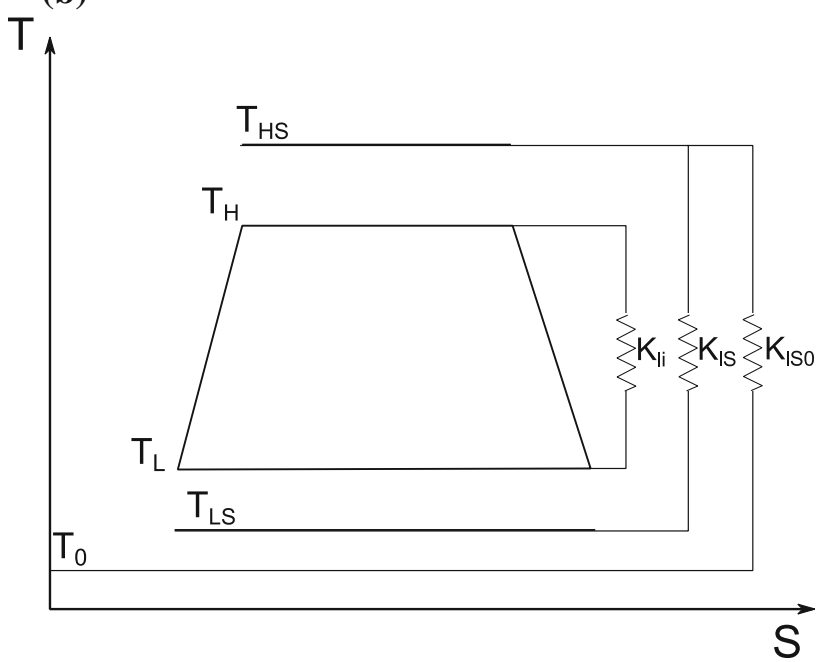

Fig. 2 a Scheme and b entropy diagram of a Novikov-CurzonAhlborn converter with infinite source and sink 
Table 1 Expression of the first and second laws at different scales, the converter, the system and the system in the environment

\begin{tabular}{|c|c|c|c|c|}
\hline & Converter alone & Converter with its thermal contact & System & System in the environment \\
\hline Losses & & $\dot{Q}_{\mathrm{li}}=K_{\mathrm{li}}\left(T_{\mathrm{H}}-T_{\mathrm{L}}\right)$ & $\dot{Q}_{\mathrm{IS}}=K_{\mathrm{le}}\left(T_{\mathrm{HS}}-T_{\mathrm{LS}}\right)$ & $\dot{Q}_{\mathrm{IS} 0}=K_{\mathrm{lo}}\left(T_{\mathrm{HS}}-T_{0}\right)$ \\
\hline Input and output rate & $\left\{\begin{array}{l}\dot{Q}_{\mathrm{HC}} \\
\dot{Q}_{\mathrm{LC}}\end{array}\right.$ & $\left\{\begin{array}{l}\dot{Q}_{\mathrm{H}}=\dot{Q}_{\mathrm{HC}}+\dot{Q}_{\mathrm{li}} \\
\dot{Q}_{\mathrm{L}}=\dot{Q}_{\mathrm{LC}}-\dot{Q}_{\mathrm{li}}\end{array}\right.$ & $\left\{\begin{array}{l}\dot{Q}_{\mathrm{HS}}=\dot{Q}_{\mathrm{H}}+\dot{Q}_{\mathrm{IS}} \\
\dot{Q}_{\mathrm{LS}}=\dot{Q}_{\mathrm{L}}-\dot{Q}_{\mathrm{IS}}\end{array}\right.$ & $\left\{\begin{array}{l}\dot{Q}_{\mathrm{HS} 0}=\dot{Q}_{\mathrm{HS}}+\dot{Q}_{\mathrm{IS} 0} \\
\dot{Q}_{\mathrm{LS} 0}=\dot{Q}_{\mathrm{LS}}-\dot{Q}_{\mathrm{IS} 0}\end{array}\right.$ \\
\hline First law & $\dot{Q}_{\mathrm{HC}}+\dot{Q}_{\mathrm{LC}}+\dot{W}=0$ & $\dot{Q}_{\mathrm{H}}+\dot{Q}_{\mathrm{L}}+\dot{W}=0$ & $\dot{Q}_{\mathrm{HS}}+\dot{Q}_{\mathrm{LS}}+\dot{W}=0$ & $\dot{Q}_{\mathrm{HS} 0}+\dot{Q}_{\mathrm{LS} 0}+\dot{W}=0$ \\
\hline Second law & $\frac{\dot{Q}_{\mathrm{HC}}}{T_{\mathrm{H}}}+\frac{\dot{Q}_{\mathrm{LC}}}{T_{\mathrm{L}}}+\dot{S}_{\mathrm{C}}\left(T_{\mathrm{H}}, T_{\mathrm{L}}\right)=0$ & $\frac{\dot{Q}_{\mathrm{H}}}{T_{\mathrm{H}}}+\frac{\dot{Q}_{\mathrm{L}}}{T_{\mathrm{L}}}+\dot{S}_{\mathrm{i}}=0$ & $\frac{\dot{Q}_{\mathrm{HS}}}{T_{\mathrm{HS}}}+\frac{\dot{Q}_{\mathrm{LS}}}{T_{\mathrm{LS}}}+\dot{S}_{\mathrm{S}}=0$ & $\frac{\dot{Q}_{\mathrm{HS} 0}}{T_{\mathrm{HS}}}+\frac{\dot{Q}_{\mathrm{LS} 0}}{T_{0}}+\dot{S}_{0}=0$ \\
\hline
\end{tabular}

the converter internal irreversibilities and various thermal losses into account. The steady state hypothesis is used.

The cycled fluid is in contact with two infinite thermal capacities, the hot source temperature $T_{\mathrm{HS}}$ and the cold sink temperature $T_{\mathrm{LS}}$. The corresponding cycle is represented in Fig. 2b. We see on this $(T, S)$ diagram that exchangers of heat occur at constant temperatures, as it is the rule for a Carnot cycle. The cycled fluid is at $T_{\mathrm{H}}$ at the hot side and at $T_{\mathrm{L}}$ at the cold side.

Consequently, the converter alone is subject to an entering heat flow rate $\dot{Q}_{\mathrm{HC}}$, an outgoing heat flow rate $\dot{Q}_{\mathrm{LC}}$ and an outgoing mechanical power $\dot{W}$. If the system considered here integrates not only the converter, but also two heat exchangers with uniform external fluid temperatures equal now to $T_{\mathrm{HS}}$ and $T_{\mathrm{LS}}$, the entering heat flow rate is $\dot{Q}_{\mathrm{H}}$ and the outgoing flow rate is $\dot{Q}_{\mathrm{L}}$. These two rates are affected by the converter internal heat loss $\dot{Q}_{l i}$, depending on $T_{\mathrm{H}}, T_{\mathrm{L}}$ and $K_{\mathrm{li}}$, the internal heat loss conductance. If we consider the thermomechanical system (converter + heat source + heat sink), the entering heat flow becomes $\dot{Q}_{\mathrm{HS}}$ and the outgoing heat flow $\dot{Q}_{\mathrm{LS}}$. The thermal heat losses occurring between the heat source and the cold sink is $\dot{Q}_{\mathrm{IS}}$ and depend on $T_{\mathrm{HS}}, T_{\mathrm{LS}}$ and $K_{\mathrm{IS}}$.

The environment is characterized by temperature $T_{0}$, that could be different from $T_{\mathrm{LS}}$. This allows to define a new assembly consisting of the system in the environment. The corresponding heat rate becomes $\dot{Q}_{\mathrm{HS} 0}$ and $\dot{Q}_{\mathrm{LS} 0}$. The complementary heat losses $\dot{Q}_{\mathrm{IS} 0}$ are dependent on $T_{\mathrm{HS}}, T_{0}$ and the corresponding heat loss conductance $K_{1 \mathrm{SO}}$.

The hypothesis of infinite heat source and cold sink is consistent with the modeling of heat transfer using lumped thermal conductances $K_{\mathrm{H}}$ and $K_{\mathrm{L}}$, such as:

$\dot{Q}_{\mathrm{H}}=K_{\mathrm{H}}\left(T_{\mathrm{HS}}-T_{\mathrm{H}}\right)$

$\dot{Q}_{\mathrm{L}}=K_{\mathrm{L}}\left(T_{\mathrm{LS}}-T_{\mathrm{L}}\right)$.

The thermodynamics sign convention implies that $\dot{Q}_{\mathrm{H}}$ is positive and $\dot{Q}_{\mathrm{L}}$ is negative.

The different heat losses are represented as positive quantities (see Table 1 line 1) whatever the control volume.
The input and output heat flow rates are given in Table 1, for the various control volumes (line 2).

Applying the first law at different scales (Table 1 line 3) shows that the engine mechanical power remains the same whatever the control volume. However, the entropy balances (Table 1 line 4 ) are different at the various scales and depend fundamentally on $\dot{S}_{\mathrm{C}}$, the rate of entropy production inside the converter. As can be seen, $\dot{S}_{\mathrm{C}}$ is represented as a function of $T_{\mathrm{H}}$ and $T_{\mathrm{L}}$, which depend mainly of the converter quality.

\section{Multiobjective optimization}

Definition of the multiobjective optimization function

The control volume considered in this new objective function is the system in the environment. This function combines the maximization of the power (MAX $|\dot{W}|)$, the minimization of the heat expanses $\left(\min \dot{Q}_{\mathrm{HS} 0}\right)$, the minimization of the rejected heat flow rate $\left(\min \left|\dot{Q}_{\mathrm{LS} 0}\right|\right)$ and the minimization of the rate of entropy production $\left(\min \dot{S}_{0}\right)$.

These four criteria are related in the multiobjective function $\mathrm{OF}_{\mathrm{S} 0}$ through weighting factors such that:

$\mathrm{OF}_{\mathrm{S} 0}=-\alpha \dot{W}-\beta \dot{Q}_{\mathrm{HS} 0}+\gamma \dot{Q}_{\mathrm{LS} 0}-\delta T_{0} \dot{S}_{0}$

with:

$\alpha+\beta+\gamma+\delta=1$.

After some calculations, we get:

$\mathrm{OF}_{\mathrm{S} 0}=\left(\alpha-\beta+\frac{T_{0}}{T_{\mathrm{HS}}} \delta\right) \dot{Q}_{\mathrm{HS} 0}+(\alpha+\gamma+\delta) \dot{Q}_{\mathrm{LS} 0}$.

As $\dot{Q}_{1 \mathrm{~S}}$ and $\dot{Q}_{\mathrm{IS} 0}$ are independent of the variables $T_{\mathrm{H}}$ and $T_{\mathrm{L}}$, this objective function is equivalent to:

$\mathrm{OF}_{\mathrm{S} 0}=\left(\alpha-\beta+\frac{T_{0}}{T_{H S}} \delta\right) \dot{Q}_{H}+(\alpha+\gamma+\delta) \dot{Q}_{L}$.

Remarks:

- If we consider the system alone (Table 1 row 3), the objective function $\mathrm{OF}_{\mathrm{S}}$ is different: 
$\mathrm{OF}_{\mathrm{S}}=\left(\alpha-\beta+\frac{T_{\mathrm{LS}}}{T_{\mathrm{HS}}} \delta\right) \dot{Q}_{\mathrm{H}}+(\alpha+\gamma+\delta) \dot{Q}_{\mathrm{L}}$

- For the converter with the two thermal contacts (Table 1 row 2), it stems from the corresponding objective function $O F_{\mathrm{i}}$ :

$\mathrm{OF}_{\mathrm{i}}=\left(\alpha-\beta+\frac{T_{\mathrm{L}}}{T_{\mathrm{H}}} \delta\right) \dot{Q}_{\mathrm{H}}+(\alpha+\gamma+\delta) \dot{Q}_{\mathrm{L}}$.

The maximization of $\mathrm{OF}_{\mathrm{S} 0}$ with respect to the two variables $T_{\mathrm{H}}$ and $T_{\mathrm{L}}$ is implemented using Lagrange multiplier method, because of the constraint for $T_{\mathrm{H}}$ and $T_{\mathrm{L}}$ which are dependent variables through the entropy balance of the converter:

$C=\frac{\dot{Q}_{\mathrm{H}}}{T_{\mathrm{H}}}+\frac{\dot{Q}_{\mathrm{L}}}{T_{\mathrm{L}}}+K_{\mathrm{li}}\left(T_{\mathrm{H}}-T_{\mathrm{L}}\right)\left(\frac{1}{T_{\mathrm{L}}}-\frac{1}{T_{\mathrm{H}}}\right)+\dot{S}_{\mathrm{C}}\left(T_{\mathrm{H}}, T_{\mathrm{L}}\right)$

Some analytical results

Optimal analytical values of temperatures $T_{\mathrm{H}}$ and $T_{\mathrm{L}}$ when $\dot{S}_{\mathrm{C}}=$ Cst and $K_{\mathrm{li}}=0$

Generally, the solution of the two differential equations system is nonanalytic. However when the converter internal heat losses are supposed to be equal to zero, analytical results are obtained for the optimal hot and cold temperatures $T_{\mathrm{H}_{\mathrm{OF}} 0}^{*}$ and $T_{\mathrm{L}_{\mathrm{OF}} 0}^{*}$ :

$T_{\mathrm{H}_{\mathrm{OF}} 0}^{*}=\sqrt{T_{\mathrm{HS}}} \frac{K_{\mathrm{L}} \sqrt{T_{\mathrm{LS}}} \sqrt{\alpha+\gamma+\delta}+K_{\mathrm{H}} \sqrt{T_{\mathrm{HS}}} \sqrt{\alpha-\beta+\frac{T_{0}}{T_{\mathrm{HS}}} \delta}}{\sqrt{\alpha-\beta+\frac{T_{0}}{T_{\mathrm{HS}}}}\left(K_{\mathrm{H}}+K_{\mathrm{L}}-\dot{S}_{\mathrm{C}}\right)}$

$T_{\mathrm{L}_{\mathrm{OF}} 0}^{*}=\sqrt{T_{\mathrm{LS}}} \frac{K_{\mathrm{L}} \sqrt{T_{\mathrm{LS}}} \sqrt{\alpha+\gamma+\delta}+K_{\mathrm{H}} \sqrt{T_{\mathrm{HS}}} \sqrt{\alpha-\beta+\frac{T_{0}}{T_{\mathrm{HS}}}}}{\sqrt{\alpha+\gamma+\delta}\left(K_{\mathrm{H}}+K_{\mathrm{L}}-\dot{S}_{\mathrm{C}}\right)}$.

Fig. 3 Case where $\delta=0$ and $\alpha$ variation for different $\beta$ values: a optimal temperatures, b mechanical power, $\mathbf{c}$ first law efficiency, $\mathbf{d}$ entropy creation rate, $\mathbf{e}$ heat rate expense at the hot side and $\mathbf{f}$ heat flow rate rejected at the cold side
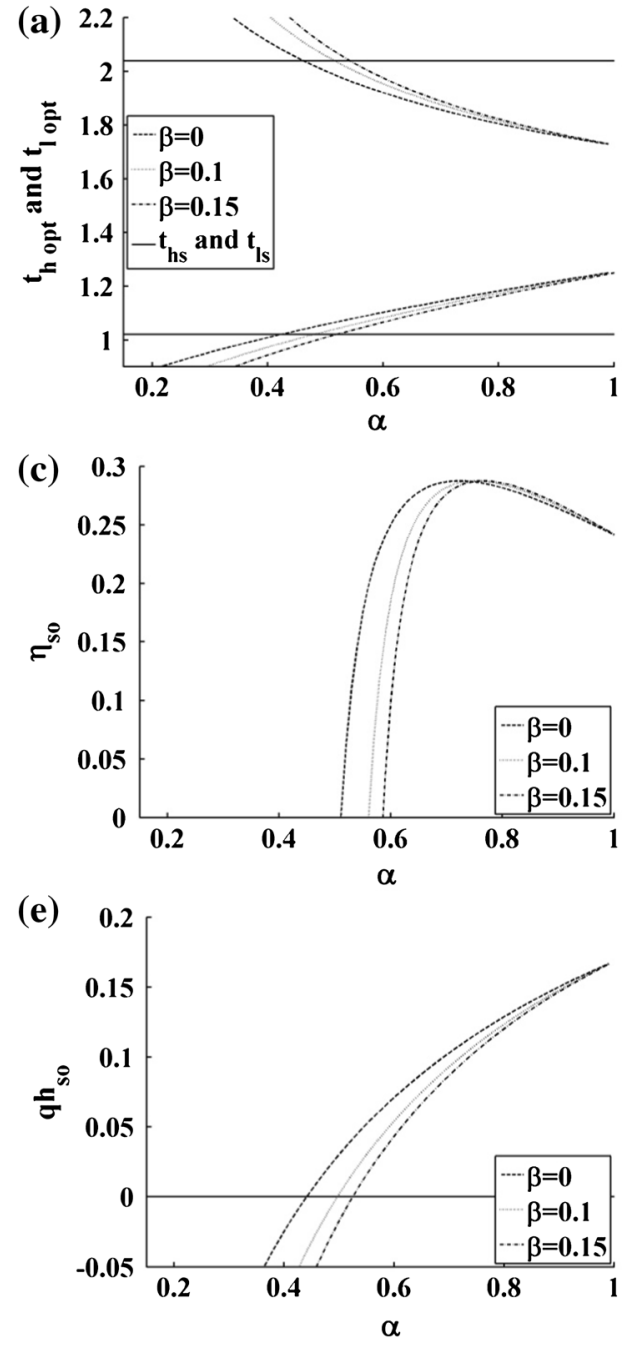
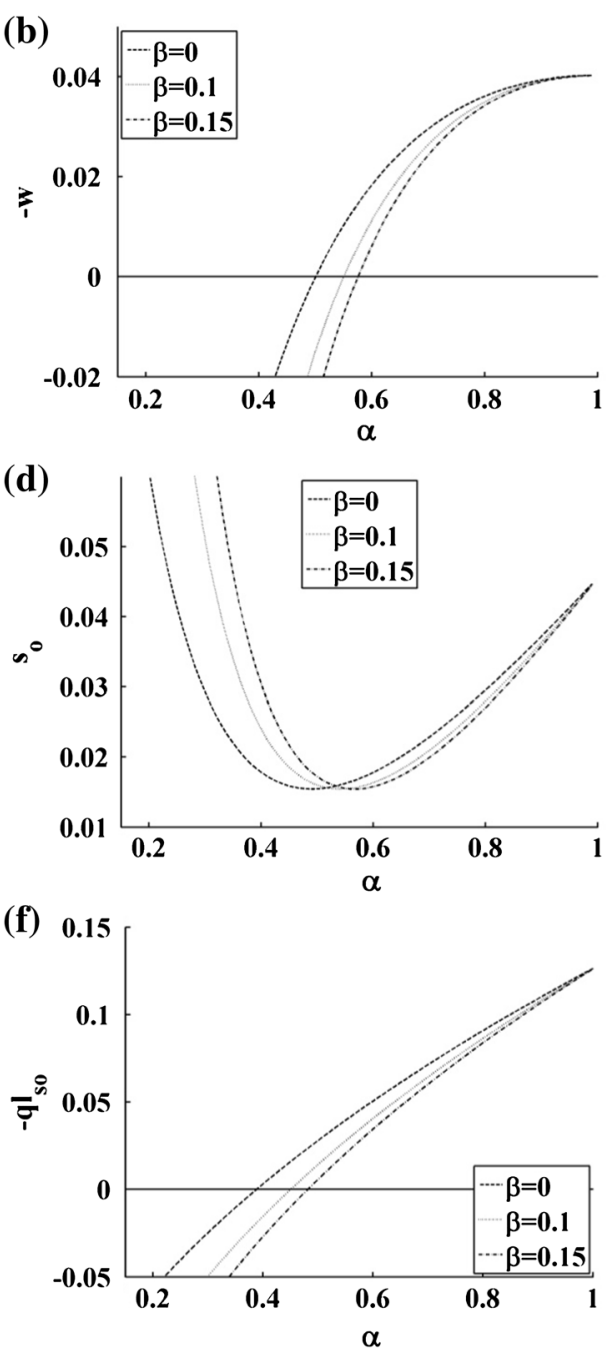
Limit case when $\alpha=1$

This case corresponds to MAX $|\dot{W}|$. The known results are retrieved:

$$
\begin{aligned}
& T_{\mathrm{H}_{\mathrm{OF} S}}^{*}(\alpha=1)=\sqrt{T_{\mathrm{HS}}} \frac{K_{\mathrm{L}} \sqrt{T_{\mathrm{LS}}}+K_{\mathrm{H}} \sqrt{T_{\mathrm{HS}}}}{K_{\mathrm{H}}+K_{\mathrm{L}}-\dot{S}_{\mathrm{C}}}<T_{\mathrm{HS}} \\
& T_{\mathrm{L}_{50}}^{*}(\alpha=1)=\sqrt{T_{\mathrm{LS}}} \frac{K_{\mathrm{L}} \sqrt{T_{\mathrm{LS}}}+K_{\mathrm{H}} \sqrt{T_{\mathrm{HS}}}}{K_{\mathrm{H}}+K_{\mathrm{L}}-\dot{S}_{\mathrm{C}}}>T_{\mathrm{LS}} .
\end{aligned}
$$

The corresponding maximum power of the engine is given by:

$$
\begin{aligned}
-\dot{W}_{\mathrm{OF}_{50}}(\alpha=1) & =\operatorname{MAX}(-\dot{W}) \\
& =\frac{K_{\mathrm{H}} K_{\mathrm{L}}\left(\sqrt{T_{\mathrm{HS}}}-\sqrt{T_{\mathrm{LS}}}\right)^{2}-\dot{S}_{\mathrm{C}}\left(K_{\mathrm{H}} T_{\mathrm{HS}}+K_{\mathrm{L}} T_{\mathrm{LS}}\right)}{K_{\mathrm{H}}+K_{\mathrm{L}}-\dot{S}_{\mathrm{c}}} .
\end{aligned}
$$

Limit case when $\beta=1$

This case corresponds to $\min \dot{Q}_{\mathrm{HS} 0}$, the heat expense of the engine. The high-level optimal temperature corresponds to:

$T_{\mathrm{H}_{\mathrm{OF} \mathrm{S}}}^{*}(\beta=1)=\frac{K_{\mathrm{H}} T_{\mathrm{HS}}}{K_{\mathrm{H}}+K_{\mathrm{L}}-\dot{S}_{\mathrm{C}}}$.

This is physically acceptable only in the equilibrium thermodynamics limit, i.e., when the heat conductances tend to infinity and $\dot{S}_{\mathrm{C}}$ tends to zero:

$T_{\mathrm{H}_{\mathrm{OF} 0}}^{*}(\beta=1)=T_{\mathrm{HS}}$.

The low-level optimal temperature is obtained using the converter entropy balance (constraint between $T_{\mathrm{H}}$ and $T_{\mathrm{L}}$ ) such that:

$T_{\mathrm{L}_{\mathrm{OF}} 0}^{*}(\beta=1)=\frac{K_{\mathrm{L}} T_{\mathrm{LS}}}{K_{\mathrm{L}}-\dot{S}_{\mathrm{C}}}$.
Fig. 4 Case where $\beta=0$ and $\alpha$ variation for different $\delta$ values: a optimal temperatures,

b mechanical power, $\mathbf{c}$ first law efficiency, $\mathbf{d}$ entropy production rate, e heat rate expense at the hot side and $\mathbf{f}$ heat flow rate rejected at the cold side
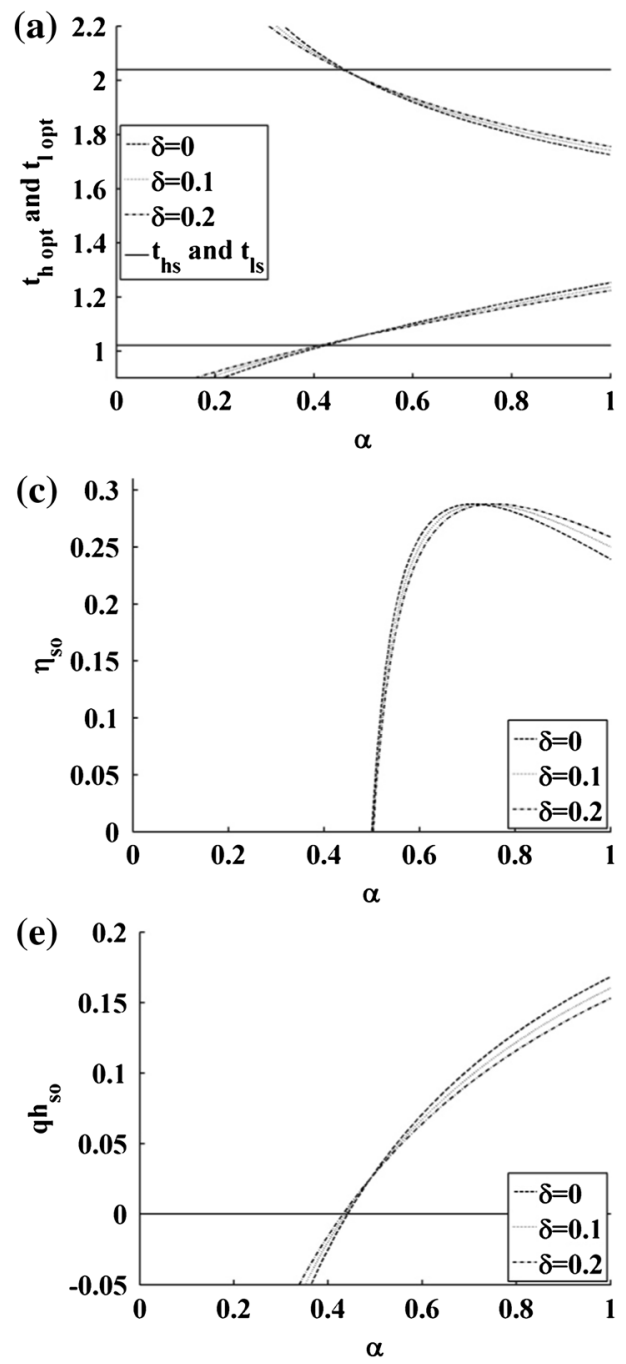
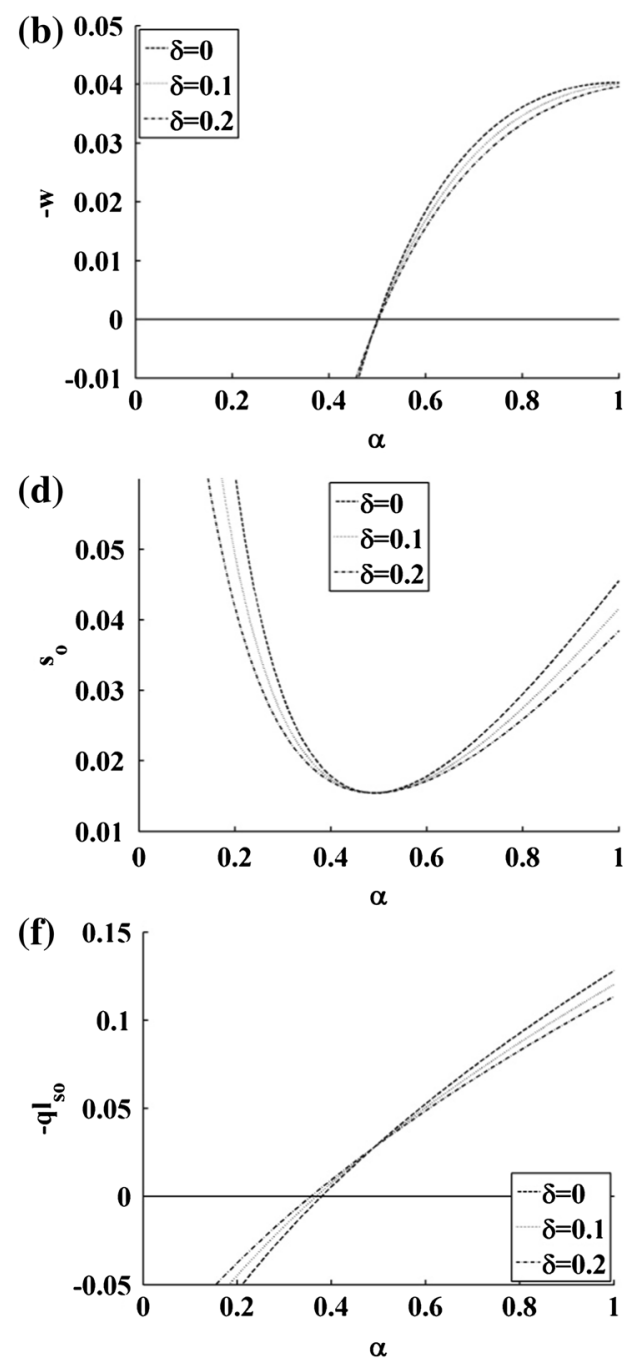
Similarly to $T_{\mathrm{H}_{\mathrm{OF} \mathrm{S}}}^{*}(\beta=1)$, this temperature must necessarily move to the equilibrium reversible situation $\left(K_{\mathrm{L}} \rightarrow \infty \dot{S}_{\mathrm{C}} \rightarrow 0\right)$ and we obtain:

$T_{\mathrm{L}_{\mathrm{OF}} 0}^{*}(\beta=1)=T_{\mathrm{LS}}$.

The associated optimum situation is such that the minimum of $\dot{Q}_{\mathrm{H}}$ is equal to zero and such is consequently the case for the corresponding engine power.

\section{Limit case when $\gamma=1$}

This case corresponds to the minimization of the rejected heat rate. Satisfaction of this objective is important because if this rejected heat rate is not used, it corresponds to heat pollution.

Getting the solution for this case is formally identical to what was made in the preceding Sect. 2.2.3. The optimal temperatures are finally the same as in (16) and (18). The $\min \left|\dot{Q}_{\mathrm{L}}\right|$ corresponds to zero and the energy power is zero (equilibrium thermodynamics limit).

Limit case when $\delta=1$

This case corresponds to the minimization of the entropy production of the system in the environment at $T_{0}\left[T_{0}\right.$ reference temperature associated to $\dot{S}_{0}$ entropy rate, as appears in (3)].

The two optimal temperatures are:

$$
\begin{gathered}
T_{\mathrm{H}_{\mathrm{OF}_{\mathrm{S}} 0}}^{*}(\delta=1)=T_{\mathrm{HS}} \frac{K_{\mathrm{L}} \sqrt{T_{\mathrm{LS}}}+K_{\mathrm{H}} \sqrt{T_{0}}}{\sqrt{T_{0}}\left(K_{\mathrm{H}}+K_{\mathrm{L}}-\dot{S}_{\mathrm{C}}\right)} \\
T_{\mathrm{L}_{\mathrm{OF}_{\mathrm{S} 0}}^{*}}(\delta=1)=T_{\mathrm{LS}} \frac{K_{\mathrm{L}} \sqrt{T_{\mathrm{LS}}}+K_{\mathrm{H}} \sqrt{T_{0}}}{\sqrt{T_{\mathrm{LS}}}\left(K_{\mathrm{H}}+K_{\mathrm{L}}-\dot{S}_{\mathrm{C}}\right)} .
\end{gathered}
$$

In fact, these mathematical results are physically acceptable only if $T_{\mathrm{LS}}=T_{0}$ and $\dot{S}_{\mathrm{C}}=0$. It corresponds to the equilibrium thermodynamics limit.
Fig. 5 Case where $\gamma=0$ and variation for different $\beta$ values: a optimal temperatures, b mechanical power, c first law efficiency, $\mathbf{d}$ entropy production rate, e heat rate expense at the hot side and $\mathbf{f}$ heat flow rate rejected at the cold side
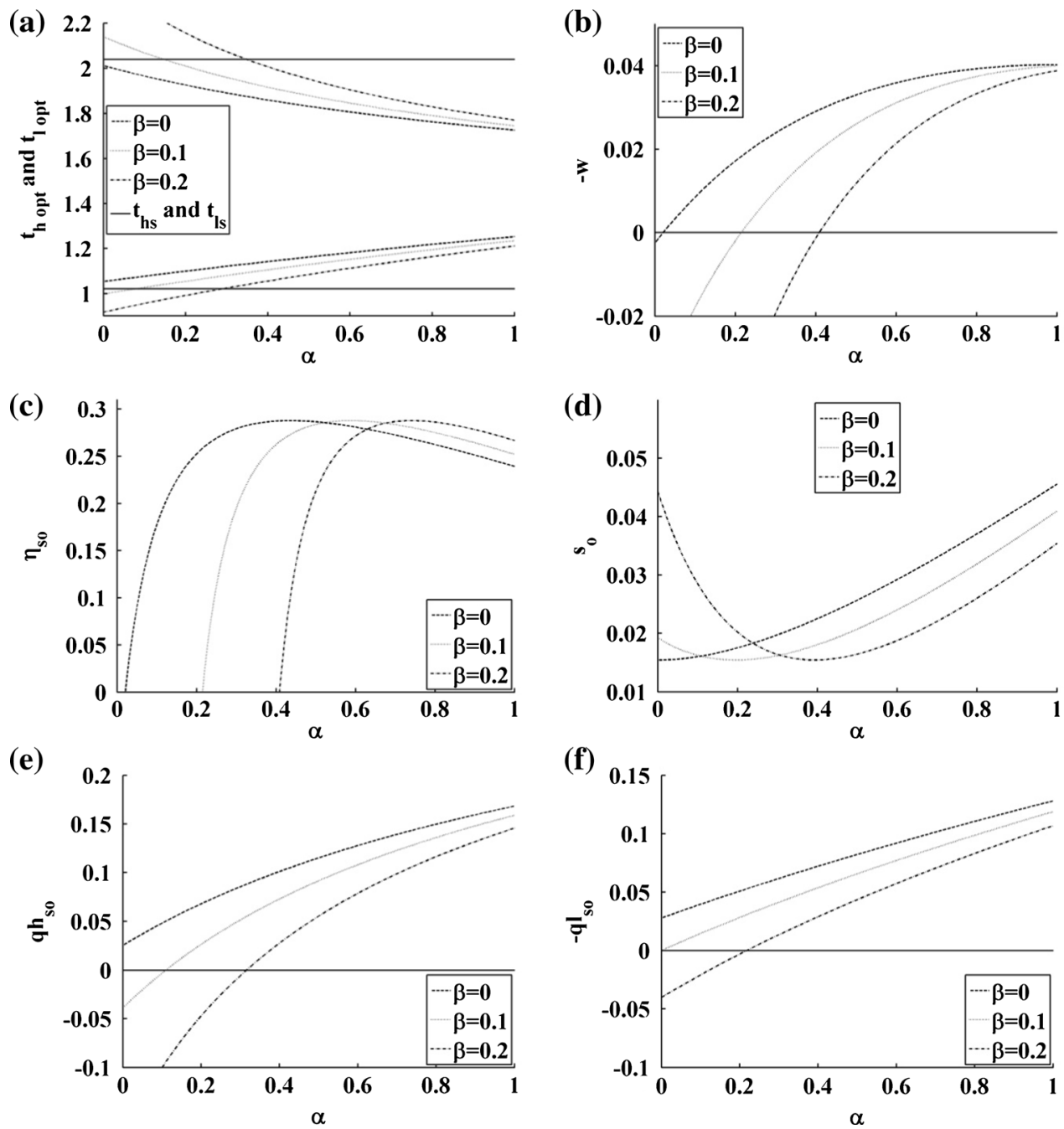

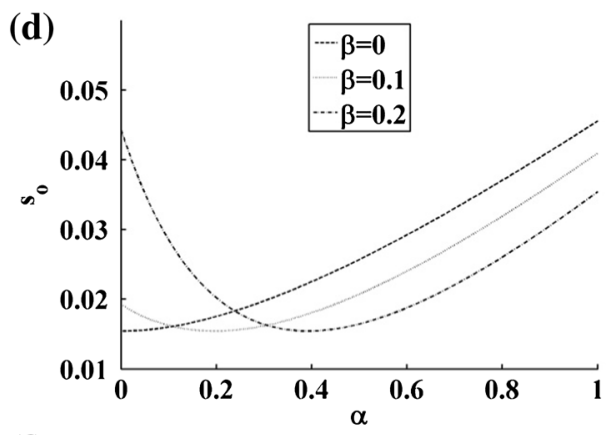

(f)

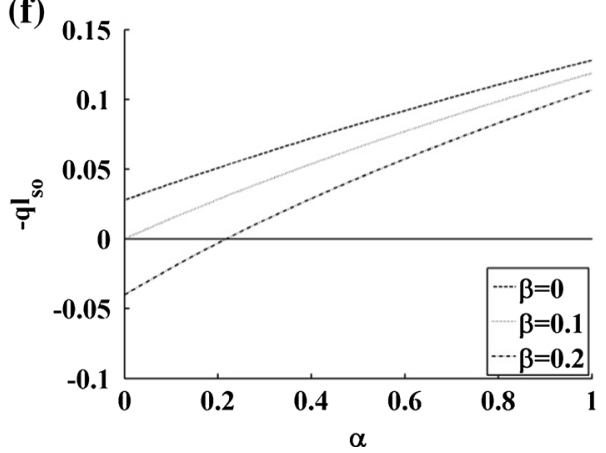




\section{Appraisal of the physical limitations regarding engine optimization}

For an engine, the weighting factors $\alpha, \beta, \gamma$ and $\delta$ are dependent of physical limitations. The first condition to satisfy is related to the hot side optimal temperature $T_{\mathrm{H}_{\mathrm{OF}} \mathrm{s}}^{*}$ :

$T_{\mathrm{H}_{\mathrm{OF} \mathrm{S} 0}}^{*}<T_{\mathrm{HS}}$.

According to the simplified analytical case (10) it comes:

$\frac{1-\beta}{\alpha-\beta+\frac{T_{0}}{T_{\mathrm{HS}}} \delta}<\frac{T_{\mathrm{HS}}}{T_{\mathrm{LS}}}\left(\frac{K_{\mathrm{L}}-\dot{S}_{\mathrm{C}}}{K_{\mathrm{L}}}\right)^{2}$.

The second condition to satisfy is related to the cold side optimal temperature $T_{\mathrm{L}_{\mathrm{F}} \mathrm{s}}^{*}$ :

$T_{\mathrm{L}_{\mathrm{OF} 0}}^{*}>T_{\mathrm{LS}}$.
This second condition becomes, according to (11), for the same analytical case:

$\frac{1-\beta}{\alpha-\beta+\frac{T_{0}}{T_{\mathrm{HS}}} \delta}<\frac{T_{\mathrm{HS}}}{T_{\mathrm{LS}}}\left(\frac{K_{\mathrm{H}}}{K_{\mathrm{H}}-\dot{S}_{\mathrm{C}}}\right)^{2}$.

Remark: It appears that the relation among $\alpha, \beta, \delta$ (and $\gamma$ ) depends on the ratio $\frac{T_{\mathrm{HS}}}{T_{\mathrm{LS}}}$, the value of $K_{\mathrm{H}}, K_{\mathrm{L}}$ and $\dot{S}_{\mathrm{C}}$.

The case where $\delta=1$ has been treaded in Sect. 2.2.5. Consequently, we focus here on the limitations regarding weighting factors $\beta$ and $\gamma$ when $\alpha$ is variable.

Limitation relative to the $\beta$ factor

It is easy to deduce from (22) the following condition relative to $\beta$ :
Fig. 6 Case where $\beta=0$ and variation for different $\gamma$ values: a optimal temperatures,

b mechanical power, c first law efficiency, $\mathbf{d}$ entropy production rate, $\mathbf{e}$ heat rate expense at the hot side and $\mathbf{f}$ heat flow rate rejected at the cold side

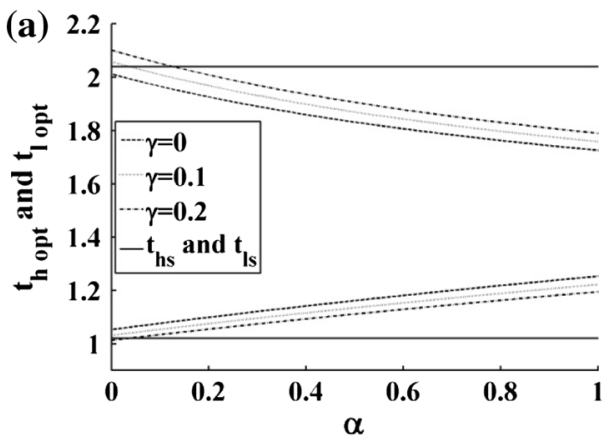

(c)

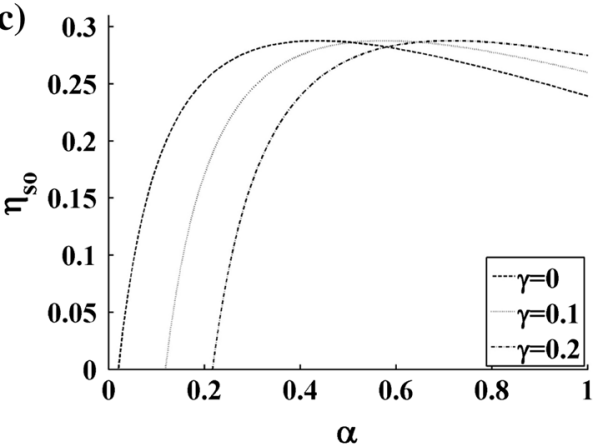

(e)

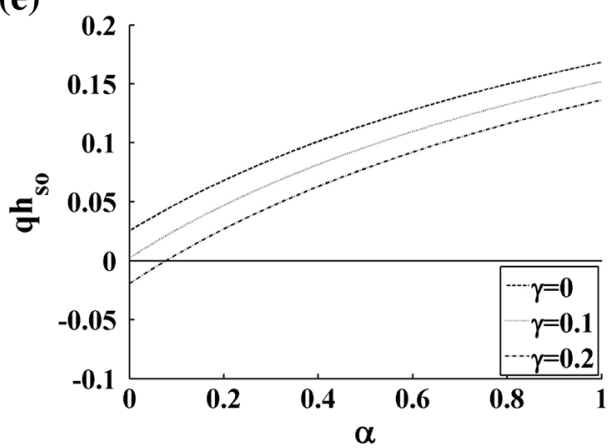

(b)

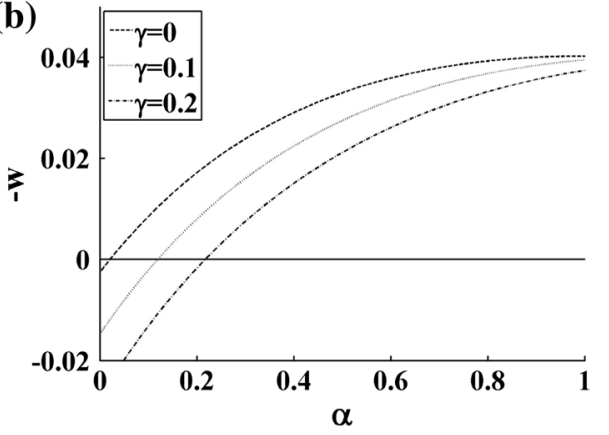

(d)

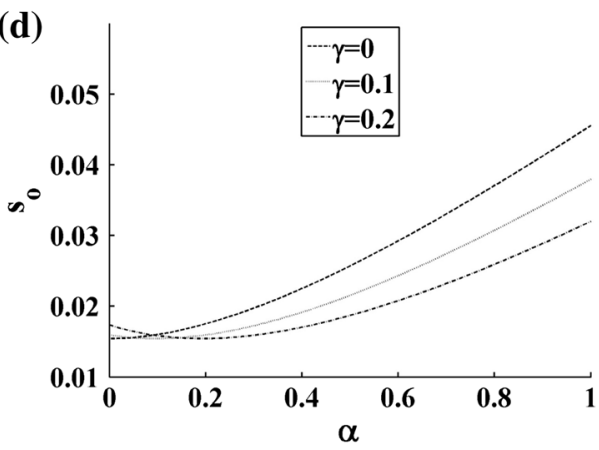

(f)

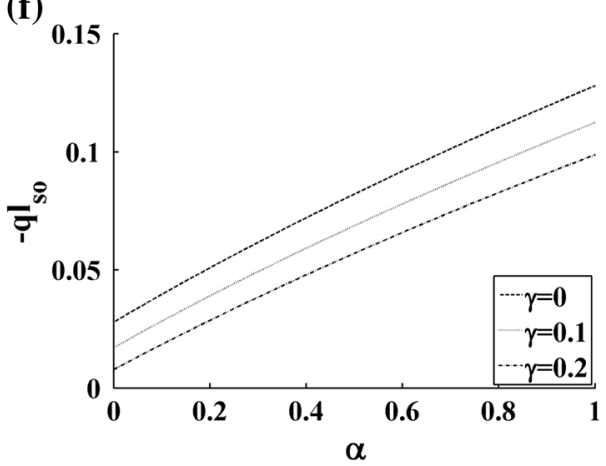


$\beta<\frac{\left(\alpha+\delta \frac{T_{0}}{T_{\mathrm{HS}}}\right)\left[\sqrt{\frac{T_{\mathrm{HS}}}{T_{\mathrm{LS}}}} \frac{K_{\mathrm{L}}-\dot{S}_{\mathrm{C}}}{K_{\mathrm{L}}}\right]^{2}-1}{\left[\sqrt{\frac{T_{\mathrm{HS}}}{T_{\mathrm{LS}}}} \frac{K_{\mathrm{L}}-\dot{S}_{\mathrm{C}}}{K_{\mathrm{L}}}\right]^{2}-1}$.

When the entropy production criterion is ignored $(\delta=0)$ and $\dot{S}_{\mathrm{C}}=0$ (endoreversible case), then:

$\beta<\frac{\alpha+\eta_{\mathrm{CS}}-1}{\eta_{\mathrm{CS}}}$,

where $\alpha$ must be chosen with a value satisfying:

$1-\eta_{\mathrm{CS}}<\alpha<1$

with $\eta_{\mathrm{CS}}=1-\frac{T_{\mathrm{LS}}}{T_{\mathrm{HS}}}$, the Carnot efficiency of the system.

The second condition to fulfill (23) gives the same limitation for the same simplified case.

Limitation relative to the $\gamma$ factor

After some calculations, the condition (21) is expressed as:

Fig. 7 Case where $\delta=0$ and variation for different $\gamma$ values: a optimal temperatures, b mechanical power, c first law efficiency, $\mathbf{d}$ entropy production rate, $\mathbf{e}$ heat rate expense at the hot side and $\mathbf{f}$ heat flow rate rejected at the cold side
$\gamma>\frac{1+(\alpha+\delta)\left[\sqrt{\frac{T_{\mathrm{LS}}}{T_{\mathrm{HS}}}} \frac{K_{\mathrm{L}}-\dot{S}_{\mathrm{C}}}{K_{\mathrm{L}}}\right]^{2}-2 \alpha-\left(1+\frac{T_{0}}{T_{\mathrm{HS}}}\right) \delta}{1-\left[\sqrt{\frac{T_{\mathrm{LS}}}{T_{\mathrm{HS}}}} \frac{K_{\mathrm{L}}-\dot{S}_{\mathrm{C}}}{K_{\mathrm{L}}}\right]^{2}}$.

The corresponding restriction for the allowed $\alpha$ values is:

$\frac{1-\eta_{\mathrm{CS}}}{1+\eta_{\mathrm{CS}}}<\alpha<\frac{1}{1+\eta_{\mathrm{CS}}}$.

Again, some calculations allow proving that the second condition (23) to fulfill gives the same domain of variation for $\alpha$.

\section{Some numerical results related to general multiobjective optimization}

In this section, some numerical results are presented for various values of the set of weighting factors $(\alpha, \beta, \gamma$ and $\delta$ ) satisfying the general physical limitations considered in Sect. 3.
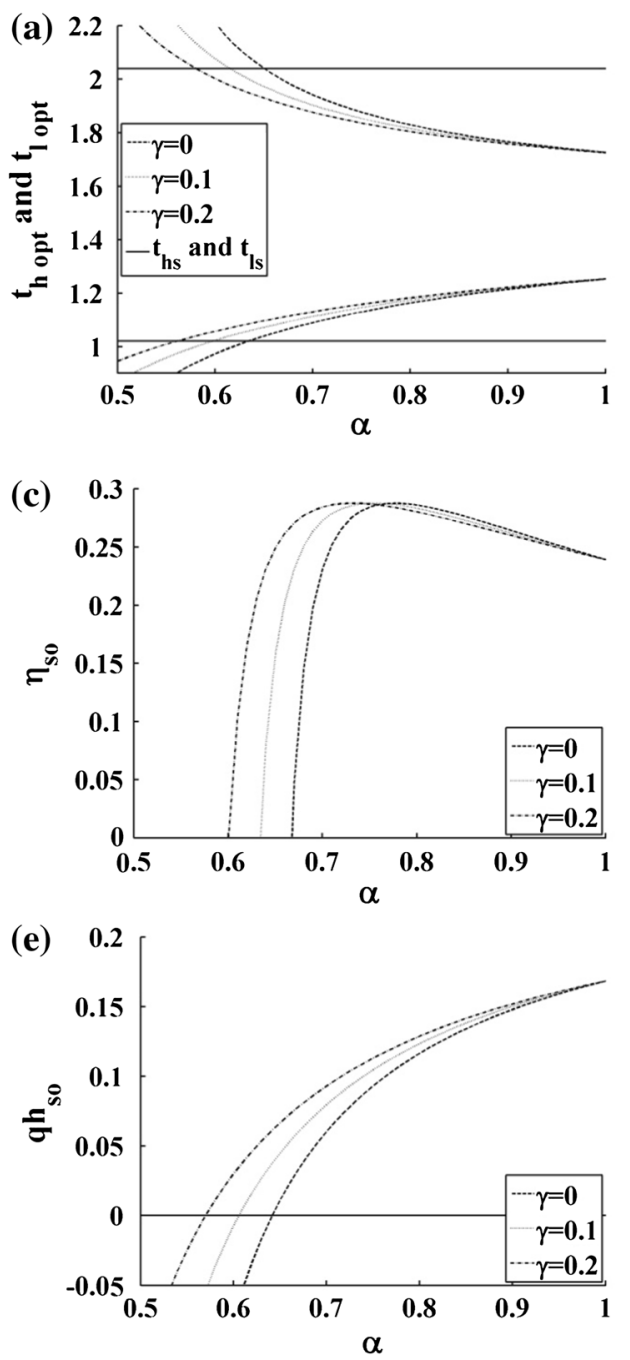
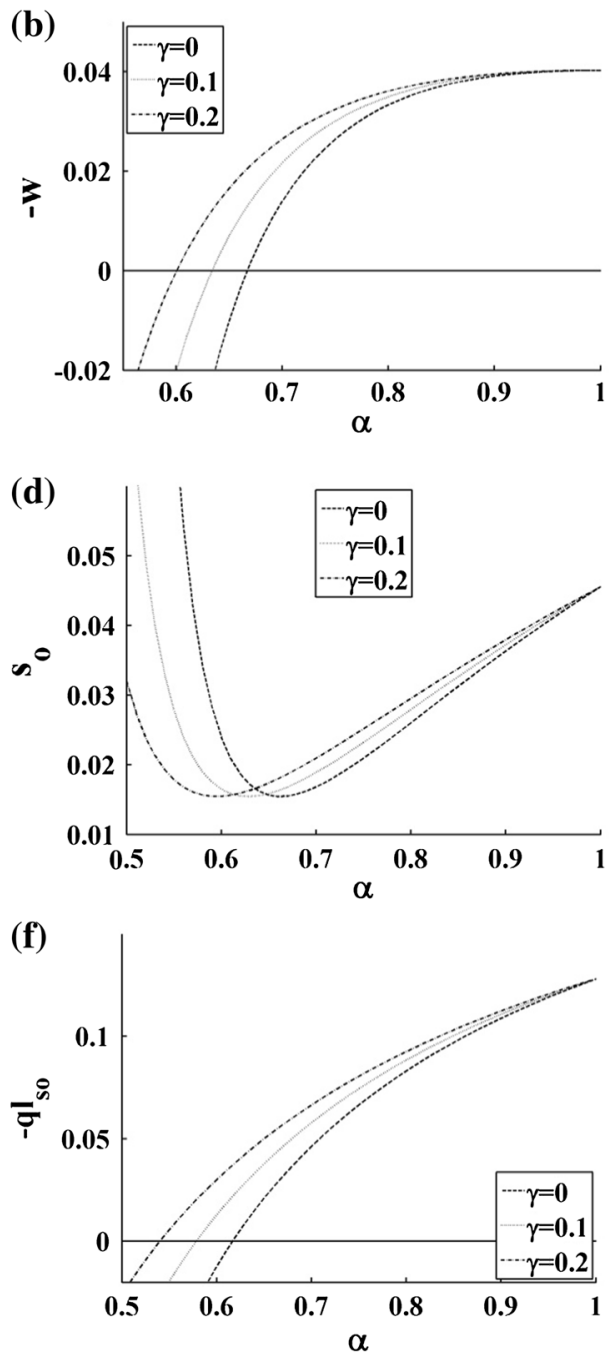
Fig. 8 Case where $\gamma=0$ and $\alpha$ variation for different $\delta$ values: a optimal temperatures,

b mechanical power, $\mathbf{c}$ first law efficiency, $\mathbf{d}$ entropy production rate, $\mathbf{e}$ heat rate expense at the hot side and $\mathbf{f}$ heat flow rate rejected at the cold side
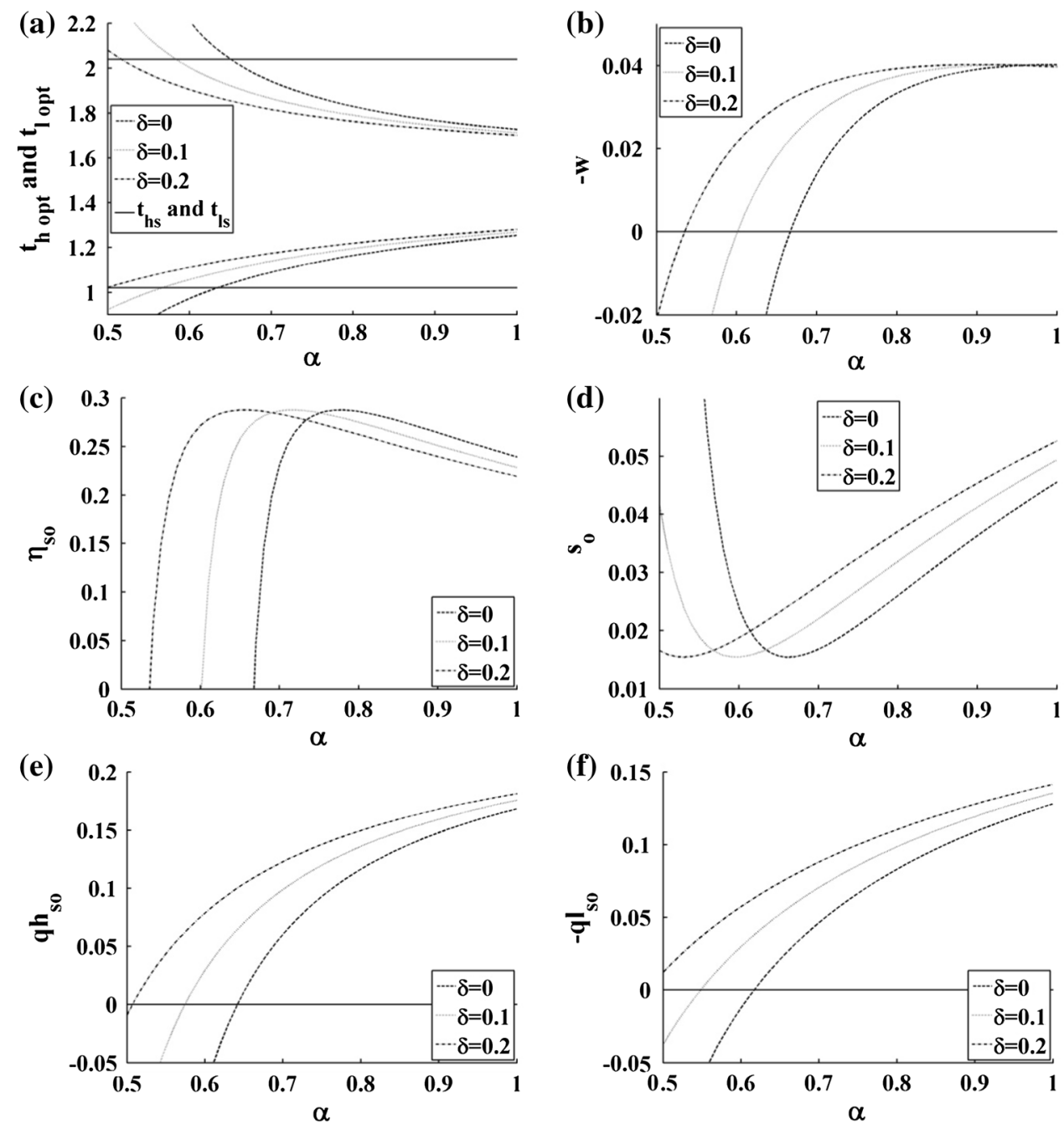

The results are presented in a dimensionless form, with the reduced variables defined hereafter:

$k_{\mathrm{j}}=\frac{K_{\mathrm{j}}}{K_{\mathrm{T}}} ; t_{\mathrm{j}}=\frac{T_{\mathrm{j}}}{T_{0}} ; s_{\mathrm{j}}=\frac{\dot{S}_{\mathrm{J}}}{K_{\mathrm{T}}} ; q_{\mathrm{j}}=\frac{\dot{Q}_{\mathrm{j}}}{K_{\mathrm{T}} T_{0}} ; w=\frac{\dot{W}}{K_{\mathrm{T}} T_{0}}$

with $K_{\mathrm{T}}=K_{\mathrm{H}}+K_{\mathrm{L}}$.

The chosen values for the parameters and for the presented results are:

$$
\begin{aligned}
& K_{\mathrm{T}}=5,000 \mathrm{~W} / \mathrm{K} ; T_{0}=293 \mathrm{~K} ; k_{\mathrm{h}}=0.5 ; k_{1}=0.5 ; s c \\
& \quad=0.0001 ; t_{\mathrm{hs}}=2.04 ; \\
& t_{\mathrm{ls}}=1.02 ; k_{\mathrm{li}}=0.05 ; k_{\mathrm{lS}}=0.01 ; k_{\mathrm{lS} 0}=0.001 .
\end{aligned}
$$

The hot side is considered to be at $600 \mathrm{~K}$ and the cold side at $300 \mathrm{~K}$, which are typical values for a Rankine cycle. The $\alpha$ weighting factor associated with the $\operatorname{MAX}|\dot{W}|$ appears as the central factor. That is why we decided to present the result through plots depending on $\alpha$ as an abscissa.
Two complementary cases are presented in Figs. 3 and 4. Figure 3 illustrates the case where $\delta=0$ for various $\beta$ values (consequently with corresponding $\gamma$ values). Figure 4 illustrates the case where $\beta=0$ for various $\delta$ values (consequently with corresponding $\gamma$ values).

Two other complementary cases are represented in Figs. 5 and 6. Figure 5 illustrates the case where $\gamma=0$ for various $\beta$ values (consequently, with corresponding $\delta$ values). Figure 6 illustrates the case where $\beta=0$, for various $\gamma$ values (consequently, corresponding $\delta$ values).

Figures 7 and 8 illustrate the last complementary couple of cases. Figure 7 illustrates the case where $\delta=0$ for various $\gamma$ values (consequently, corresponding $\beta$ values) and Fig. 8 illustrates the case where $\gamma=0$ for various $\delta$ values (consequently, with corresponding $\beta$ values).

All these curves (Figs. 3b, 4, 5, 6, 7, 8b) show that increasing $\alpha$ induces an asymptotic maximum value of the 

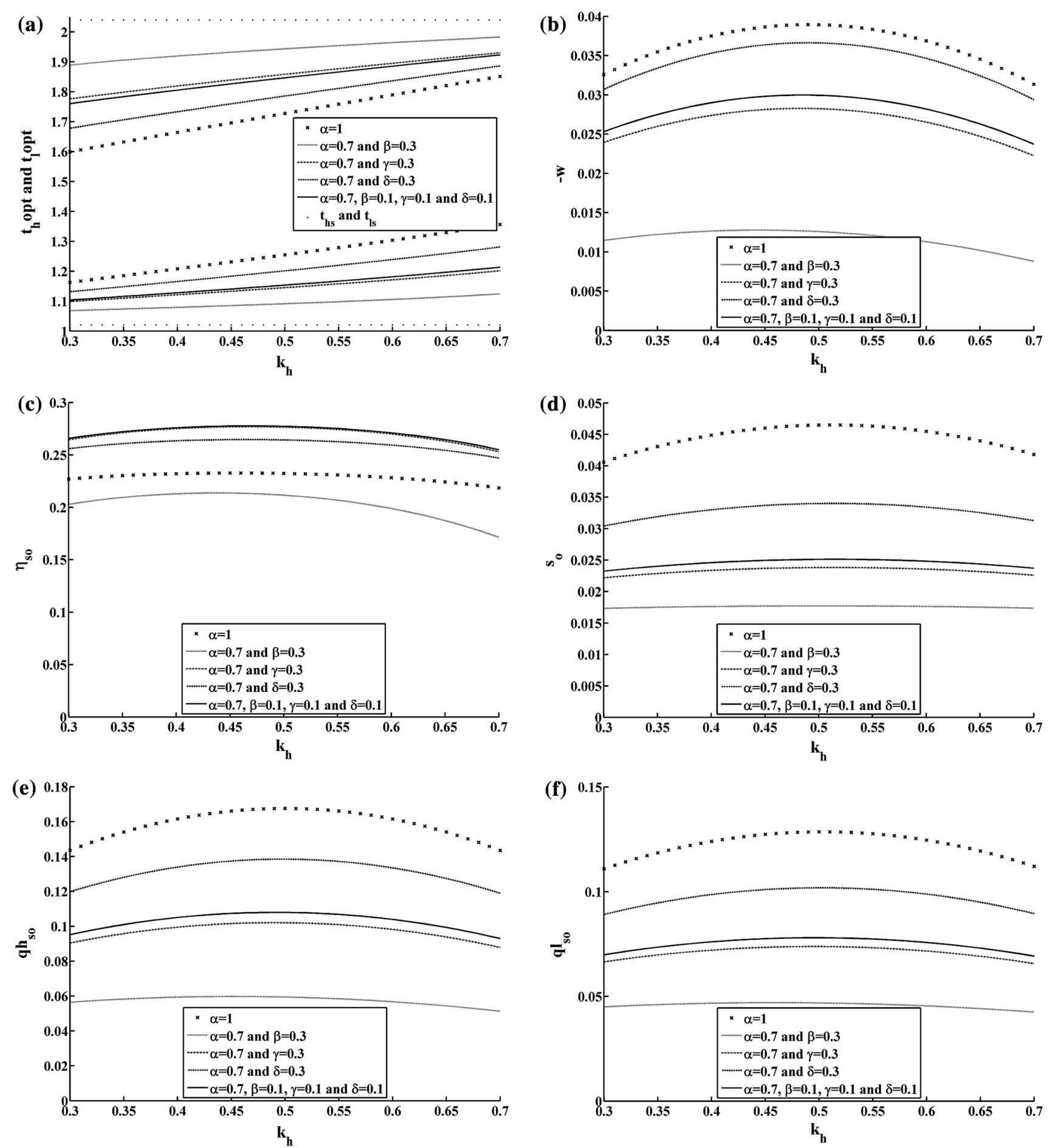

Fig. 9 Case where $k_{\mathrm{h}}$ is varied for different sets of weights $\alpha, \beta, \gamma$ and $\delta$ : a temperatures, $\mathbf{b}$ mechanical power, $\mathbf{c}$ first law efficiency, $\mathbf{d}$ entropy production rate, $\mathbf{e}$ heat rate expense at the hot side and $\mathbf{f}$ heat flow rate rejected at the cold side

engine power. This is obtained through an increase of the temperature differences between the source and converter, and sink and converter (Figs. 3a, 4, 5, 6, 7, 8a). This increasing power is related to an increase of the heat rate expense at the hot side (Figs. 3e, 4, 5, 6, 7, 8e) and an increase of the heat flow rate rejected at the cold side (Figs. 3f, 4, 5, 6, $7,8 \mathrm{f})$, as well as an increase in the entropy production for the allowed $\alpha$ values (Figs. 3d, 4, 5, 6, 7, 8d).

However, we must add that the physical limitations on $\alpha$ and $\beta$ values appear clearly for all these curves. Moreover, 
the first condition [(21) limitation at the hot side] is more restrictive for the $\alpha$ values than the second condition [(23) limitation at the cold side], see Fig. 3a. Section 3.1 and 3.2 show that the difference between these two conditions depends on the $\delta$ weighting factor and $\dot{S}_{\mathrm{C}}$, and on the entropy production in the converter. For a given $\alpha$ value, if $\beta$ increases, the heat flow rate expense (Fig. 3e) and the rejected heat rate (Fig. 3f) diminish as well as the engine power (Fig. 3b). We note that, to remain physically acceptable, the $\alpha$ value corresponding to a given $\beta$ increases with $\beta$.

The curves associated with the first law efficiency (Fig. 3c) shows that a given set of $(\alpha, \beta)$ values corresponding to the maximum efficiency exists. If $\beta$ increases, the $\alpha$ value at the optimum increases too. Accordingly, (Fig. 3d), a minimum of entropy production exists for a set of $(\alpha, \beta)$ values.

Figure 4 shows the corresponding limitations associated with the $\delta$ weighting factor. The first condition remains more restrictive than the second one, but the influence of $\delta$ is less important than the one of $\beta$ (Fig. 4a).

Whatever the case, i.e., for $\delta=0$ (Fig. 3) or for $\gamma=0$ (Fig. 5), when $\beta$ increases, the $\alpha$ limit value increases. For $\beta=0$ (Fig. 4) and $\gamma=0$ (Fig. 8), when $\delta$ increases, the $\alpha$ limit value decreases. In contrast, for $\delta=0$ (Fig. 7) when $\gamma$ increases, the $\alpha$ limit value decreases and for $\beta=0$ (Fig. 6) when $\gamma$ increases the $\alpha$ limit value increases.

\section{Conclusions and perspectives}

There are weighting thresholds related to the configuration of the system. These thresholds are function of the conductances $K_{\mathrm{H}}$ and $K_{\mathrm{L}}$, of the temperatures of source and sink $T_{\mathrm{HS}}$ and $T_{\mathrm{LS}}$ and of $\dot{S}_{\mathrm{C}}$, the rate of internal entropy creation of the converter. The different numerical results show that the factor $\beta$ is very restrictive. The minimization of the energy expenses is strongly limiting.

The optimum temperatures may differ with the optimum design. Varying transfer conductances $K_{\mathrm{H}}$ and $K_{\mathrm{L}}$ can lead to an optimum allocation of the transfer conductances. Some numerical results are presented for different sets of physically acceptable weights $\alpha, \beta, \gamma$ and $\delta$ (Fig. 9). The notations and parameters used are the same as in the previous section. The optimal mechanical power variation with $k_{\mathrm{h}}$ is plotted in Fig. 9b. The corresponding efficiency is plotted in Fig. 9c. It can be noted that a value exists for $k_{\mathrm{h}}$ that maximizes the power. There is therefore an optimal conductance distribution. A maximum efficiency can also be reached for a given value of $k_{\mathrm{h}}$. Extrema exist for the entropy production rate, heat expense and heat rejection, but they are maxima and not the goal (Fig. 9d, e, f).

Open Access This article is distributed under the terms of the Creative Commons Attribution License which permits any use, distribution, and reproduction in any medium, provided the original author(s) and the source are credited.

\section{References}

1. Chambadal, P.: Evolution et applications du concept d'entropie. Dunod, Paris (1963)

2. Chambadal, P.: Le choix du cycle thermique dans une usine génératrice nucléaire. Revue Générale d'Electricité. (1958)

3. Chambadal, P.: Les centrales nucléaires. A. Colin, Paris (1957)

4. Novikov, I.: The efficiency of atomic power stations (a review). Atomaya Energ. 3-11 (1957)

5. Curzon, F, Ahlborn, B.: Efficiency of a Carnot engine at maximum power output, Am. J. Phys. 22-24 (1975)

6. Lazzaretto, A.: Energy, economy and environment as objectives in multi-criterion optimization of thermal systems design. Energy. 1139-1157 (2001)

7. Lira-Barragan, L.: Synthesis of integrated absorption refrigeration systems involving economic and environmental objectives and quantifying social benefices. Appl. Therm. Eng. 52, 402-419 (2013)

8. Wang, J.: Multicriteria analysis of combined cooling, heating and power systems in different climate zones in China. Appl. Energy 87, 1247-1259 (2010)

9. Siitonen, S.: Implications of process energy efficiency improvements for primary energy consumption and $\mathrm{CO}_{2}$ at the national level. Appl. Energy 87, 2928-2937 (2010)

10. Sayyaadi, H.: Multi-objective optimization of a vertical ground source heat pump using evolutionnary algorithm. Energy Convers. Manag. 50, 2035-2046 (2009)

11. Ahmadi, M.: Optimal design of a solar driven heat engine based on thermal and thermoeconomic criteria. Energy Convers. Manage. (2013)

12. Angulo-Brown, F.: An ecological optimisation criterion for finitetime heat engines. J. Appl. Phys. 69, 7465-7469 (1991)

13. Yan, Z.: Comment on "An ecological optimization criterion for finite-time heat engines”. J. Appl. Phys. 73, 3583 (1993)

14. Feidt, M.: Thermodynamique et optimisation énergétique des systèmes et procédés. TEC et DOC, Paris (1996)

15. Feidt, M.: Thermodynamique optimale en dimensions physiques finies. Hermès, Paris (2013) 